\title{
Selective Antiproliferative Withanolides from Species in the Genera Eriolarynx and Deprea.
}

Sebastián J. Castro,${ }^{\dagger}$ Carina N. Casero,${ }^{\dagger}$ José M. Padrón, ${ }^{*}, \sharp$ and Viviana E. Nicotra ${ }^{*} \dagger$

† Facultad de Ciencias Químicas, Instituto Multidisciplinario de Biología Vegetal (IMBIVCONICET), Universidad Nacional de Córdoba, Casilla de Correo 495, 5000 Córdoba, Argentina

\$BioLab, Instituto Universitario de Bio-Orgánica “Antonio González” (IUBO-AG), Centro de Investigaciones Biomédicas de Canarias (CIBICAN), Universidad de La Laguna, C/Astrofísico Francisco Sánchez 2, 38206 La Laguna, Spain

\section{TABLE OF CONTENTS}

Figure S1. Relevant NOE correlations of compound 1.

Figure S2. ${ }^{1} \mathrm{H}$ NMR spectrum of compound 2.

Figure S3. COSY spectrum of compound 2.

Figure S4. HSQC spectrum of compound 2.

Figure S5. HMBC spectrum of compound 2.

Figure S6. ${ }^{1}$ H NMR spectrum of compound 3 (epimeric mixture at C-18).

Figure S7. COSY spectrum of compound 3 (epimeric mixture at C-18).

Figure S8. HSQC spectrum of compound $\mathbf{3}$ (epimeric mixture at C-18).

Figure S9. HMBC spectrum of compound 3 (epimeric mixture at C-18).

Figure S10. ${ }^{1} \mathrm{H}$ NMR spectrum of compound 4.

Figure S11. COSY spectrum of compound 4.

Figure S12. HSQC spectrum of compound 4.

Figure S13. HMBC spectrum of compound 4.

Figure S14. ${ }^{1} \mathrm{H}$ NMR spectrum of compound 5.

Figure S15. COSY spectrum of compound 5.

Figure S16. HSQC spectrum of compound 5.

Figure S17. HMBC spectrum of compound 5.

Figure S18. Relevant NOE correlations of compounds 4 and 5.

Figure S19. $\mathrm{GI}_{50}$ range plot against tested human solid cell lines and $\mathrm{GI}_{50}$ against human fibroblasts. 
Figure S1. Relevant NOE correlations of compound $1\left(\mathrm{CDCl}_{3}, 400.13 \mathrm{MHz}\right)$.
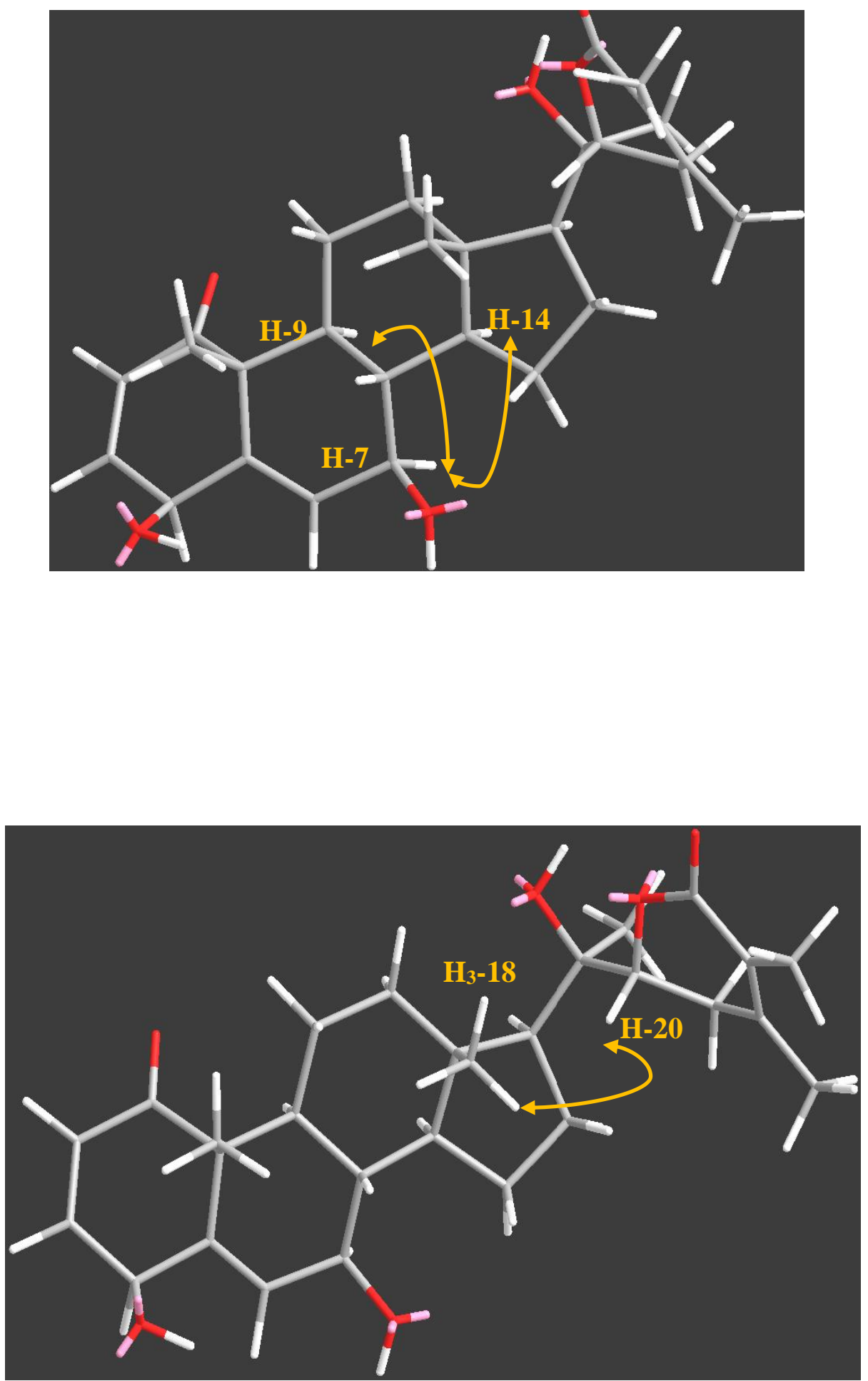
Figure S2. ${ }^{1} \mathrm{H}$ NMR spectrum of compound $2\left(\mathrm{CDCl}_{3}, 400.13 \mathrm{MHz}\right)$.

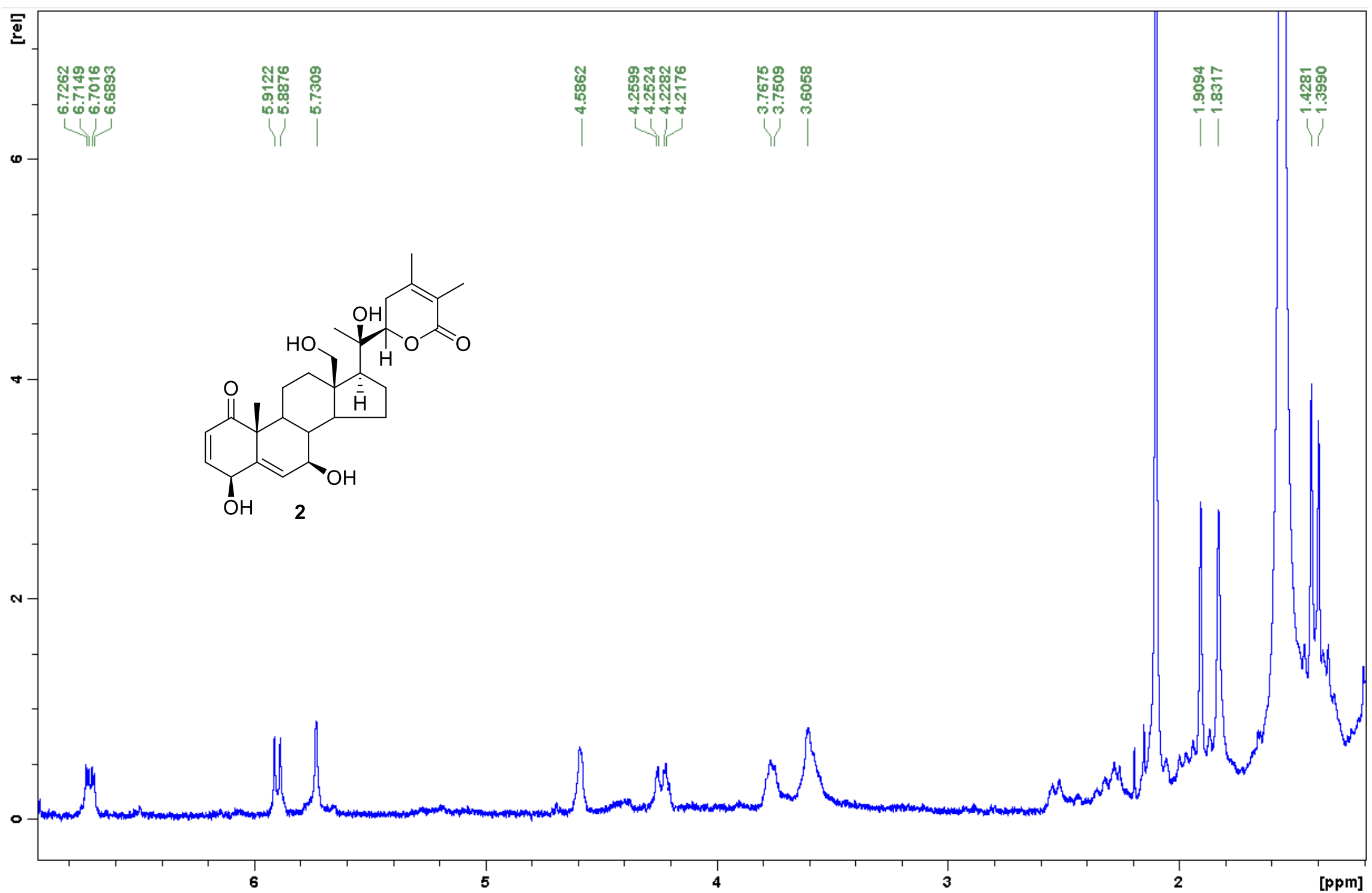


Figure S3. COSY spectrum of compound $2\left(\mathrm{CDCl}_{3}, 400.13 \mathrm{MHz}\right)$.

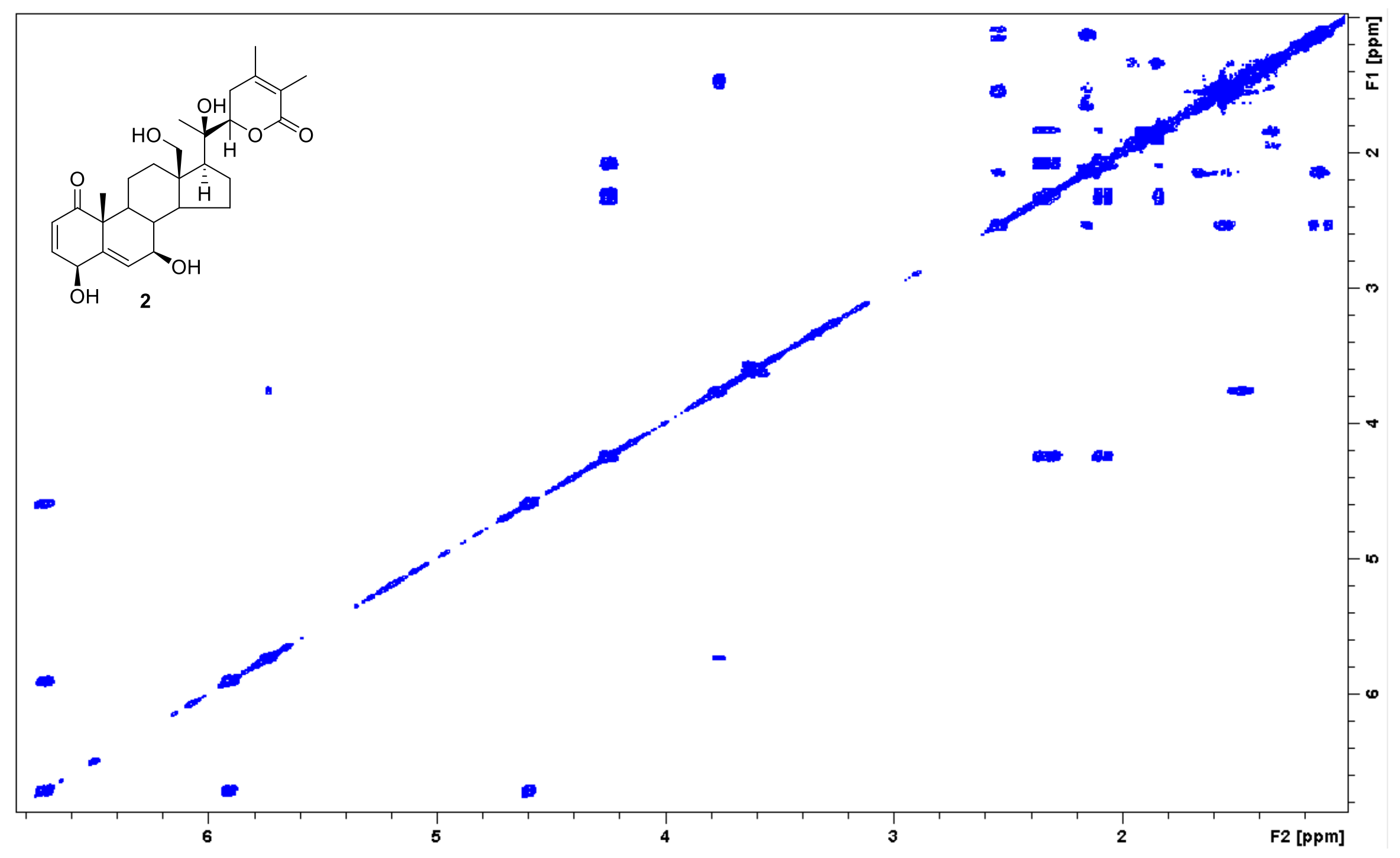


Figure S4. HSQC spectrum of compound $2\left(\mathrm{CDCl}_{3}, 400.13 \mathrm{MHz}\right)$.

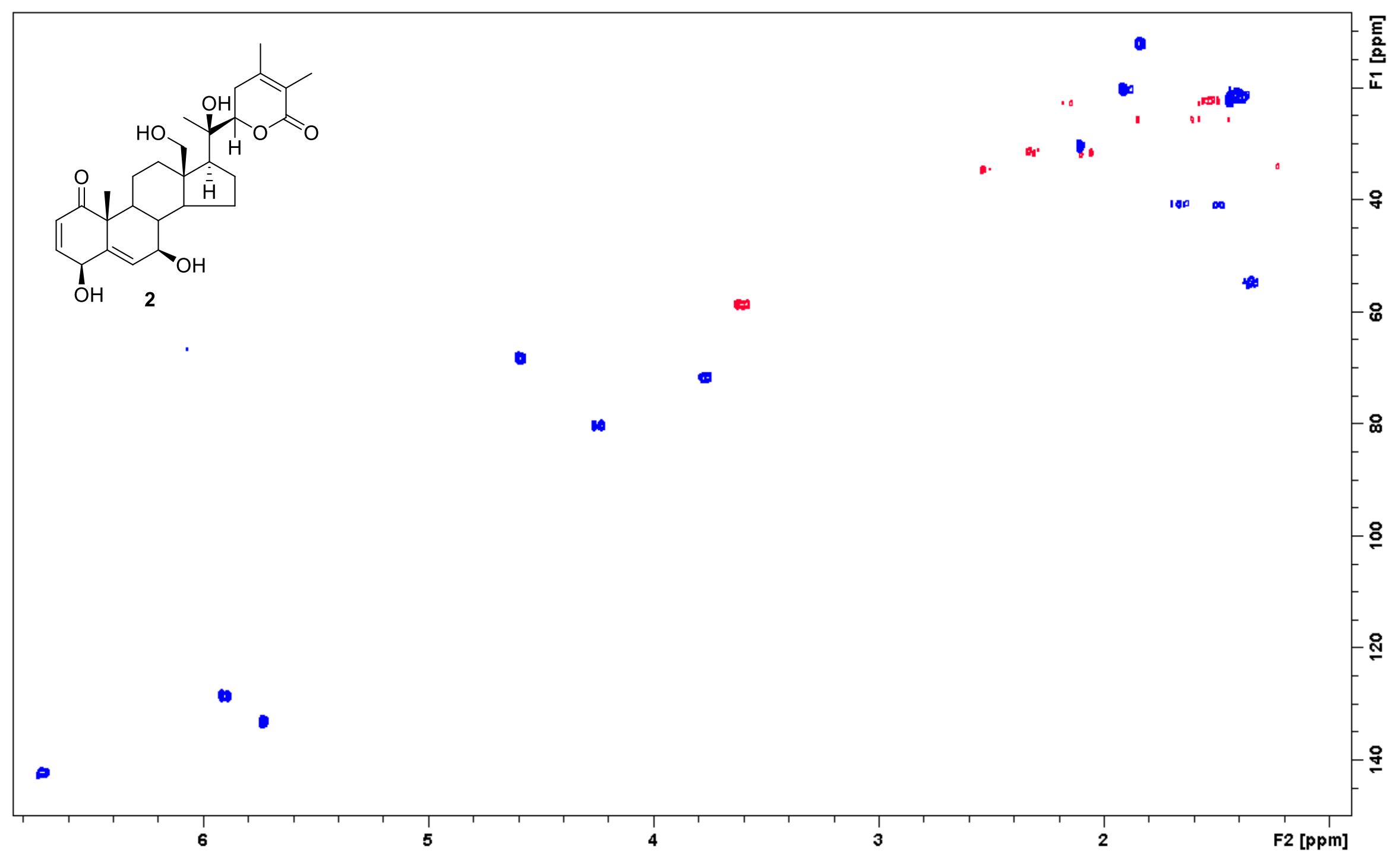


Figure S5. HMBC spectrum of compound $2\left(\mathrm{CDCl}_{3}, 400.13 \mathrm{MHz}\right)$.

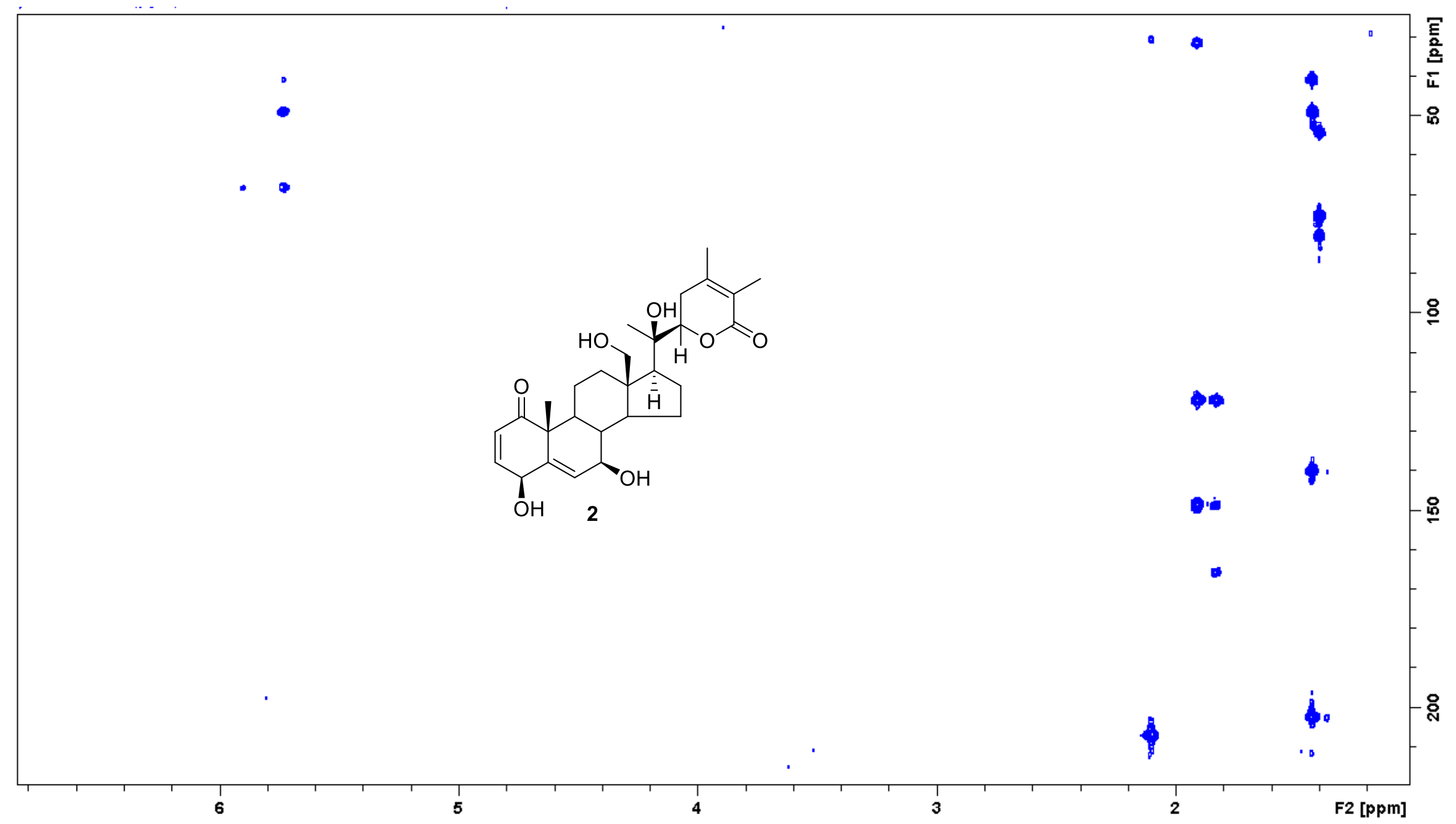


Figure S6. ${ }^{1} \mathrm{H}$ NMR spectrum of compound 3 (epimeric mixture at C-18) $\left(\mathrm{CDCl}_{3}, 400.13 \mathrm{MHz}\right)$.

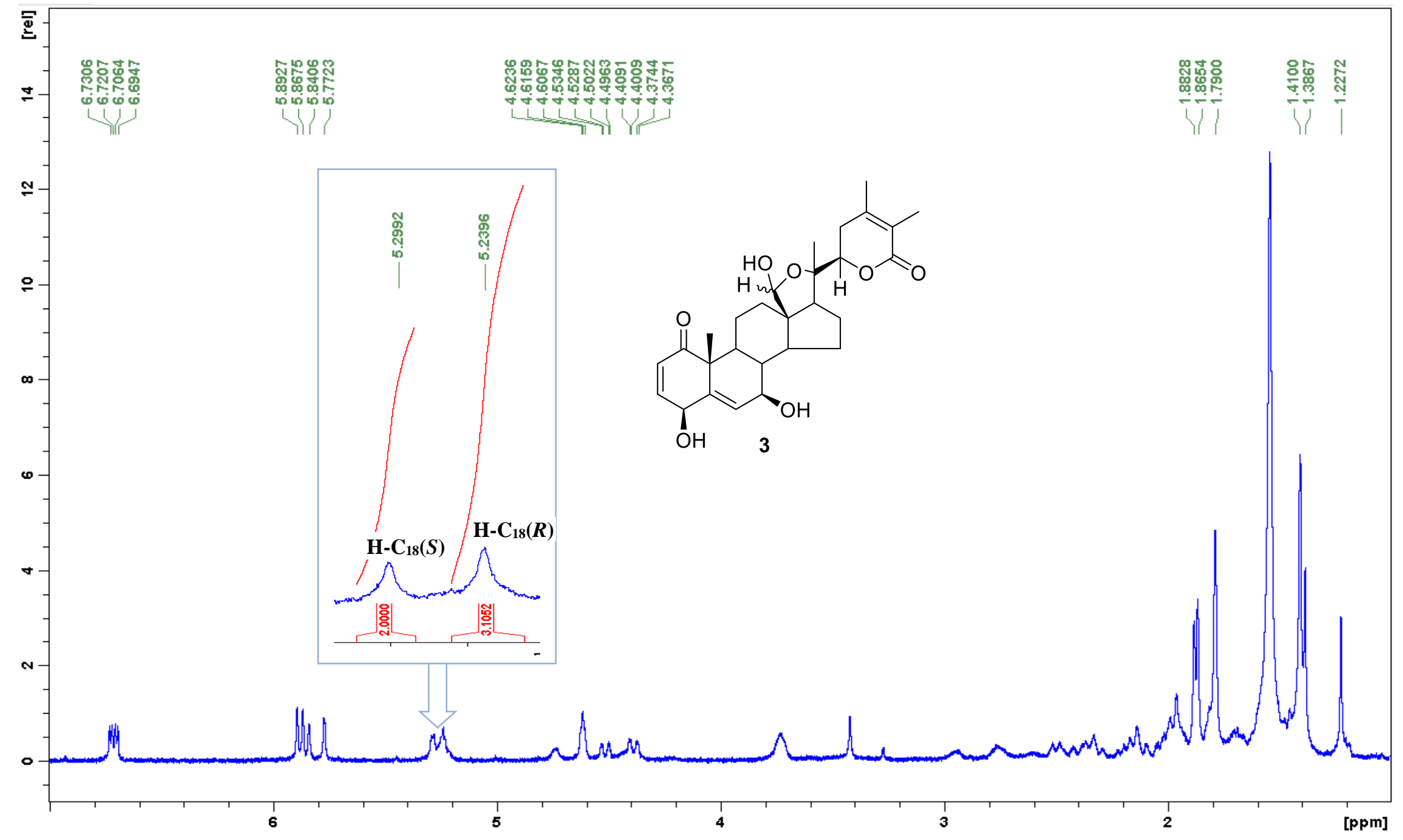


Figure S7. COSY sectrum of compound 3 (epimeric mixture at C-18) $\left(\mathrm{CDCl}_{3}, 400.13 \mathrm{MHz}\right)$.

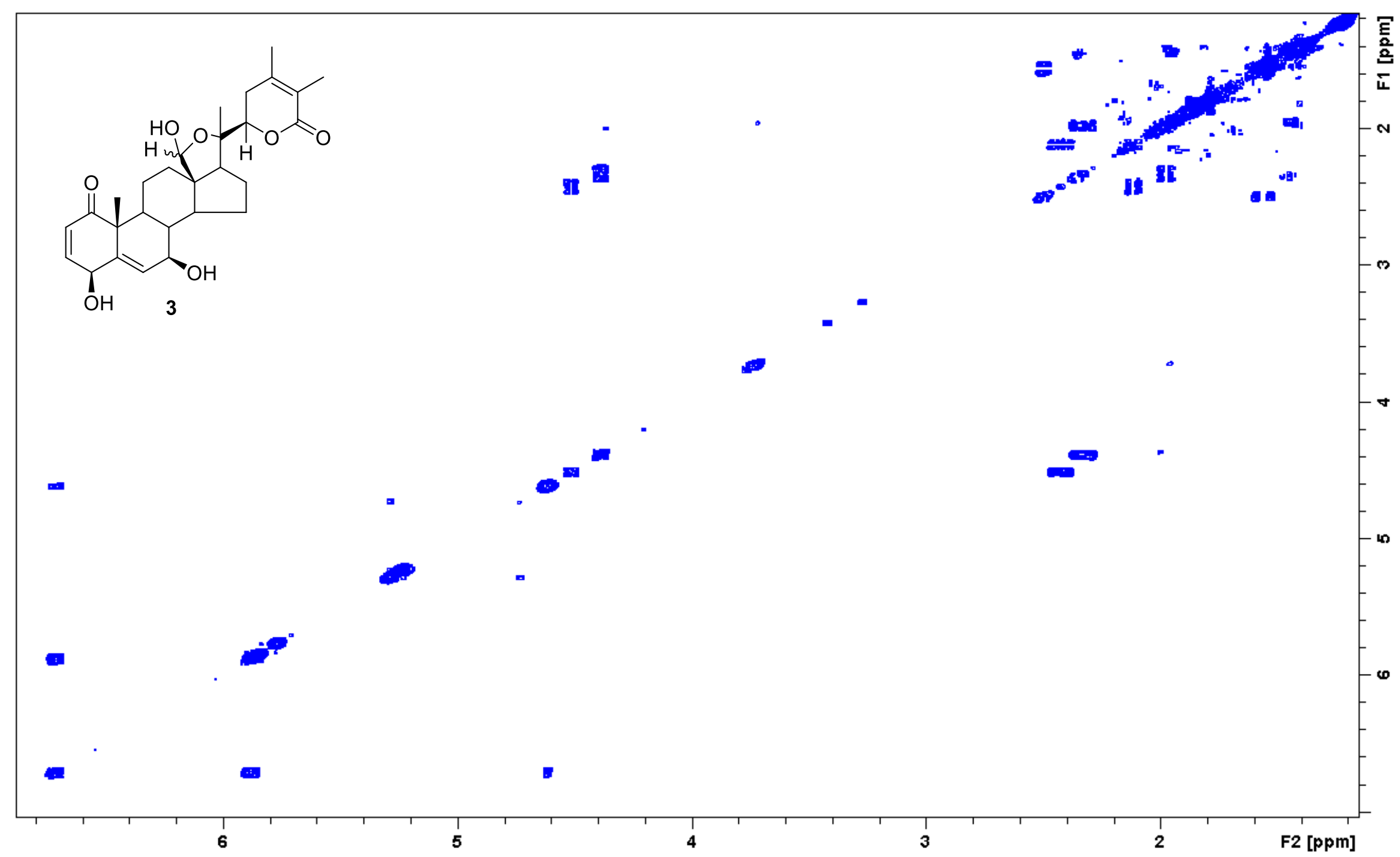


Figure S8. HSQC sectrum of compound 3 (epimeric mixture at C-18) $\left(\mathrm{CDCl}_{3}, 400.13 \mathrm{MHz}\right)$.

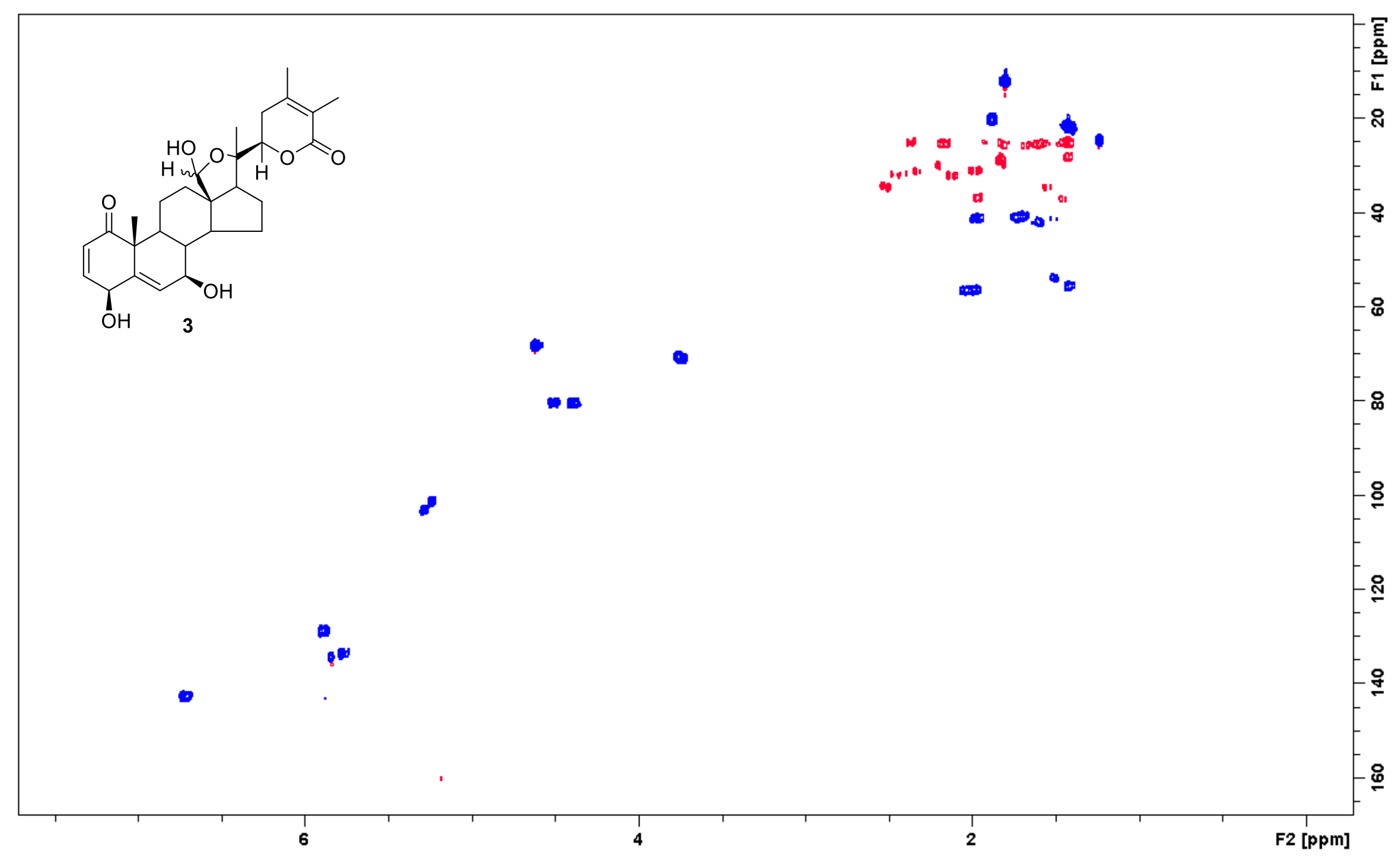


Selective Antiproliferative Withanolides from Species in the Genera Eriolarynx and Deprea. Castro et al.

Figure S9. HMBC sectrum of compound 3 (epimeric mixture at C-18) $\left(\mathrm{CDCl}_{3}, 400.13 \mathrm{MHz}\right)$.

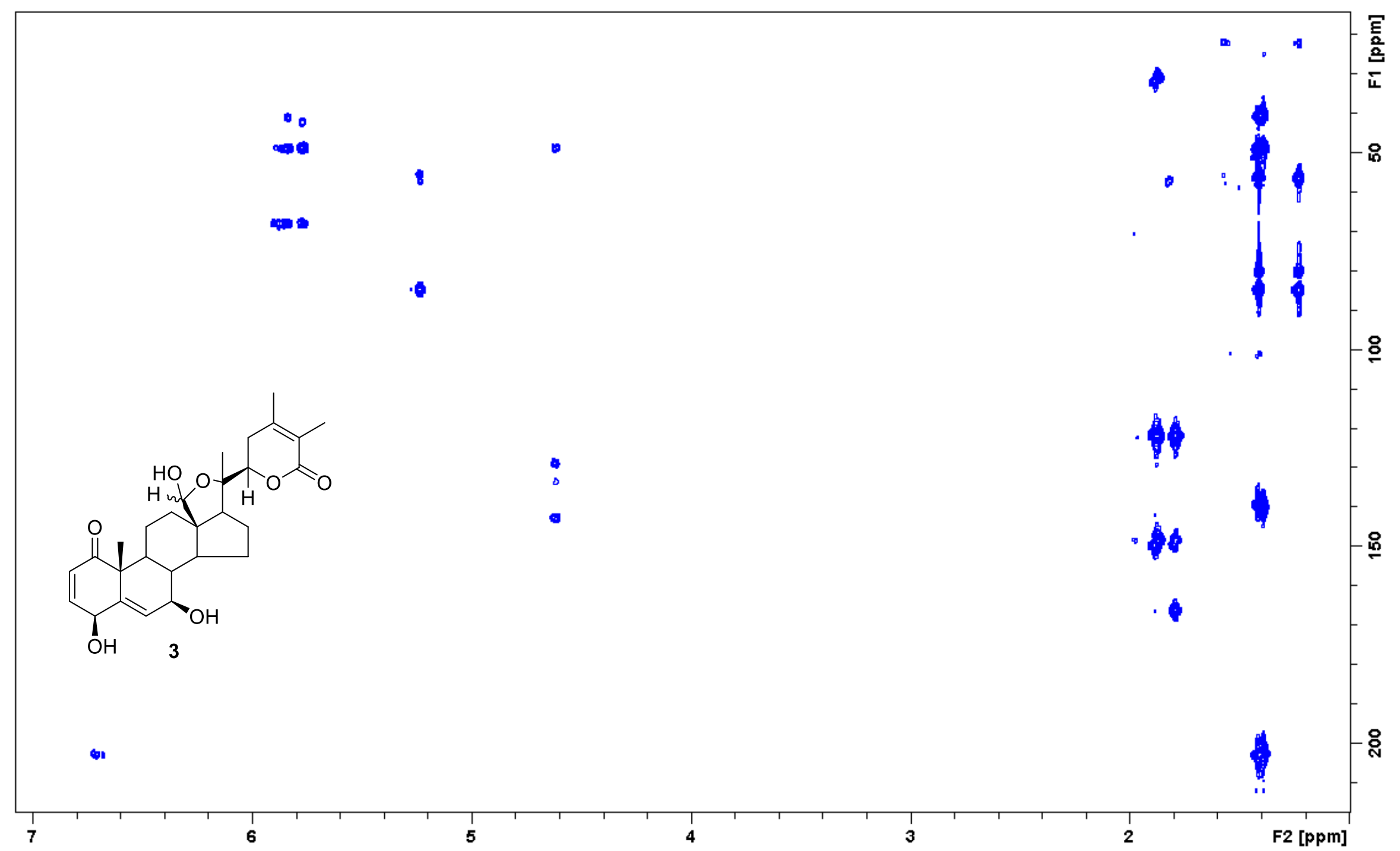


Figure S10. ${ }^{1} \mathrm{H}$ RMN spectrum of compound $4\left(\mathrm{CDCl}_{3}, 400.13 \mathrm{MHz}\right)$.

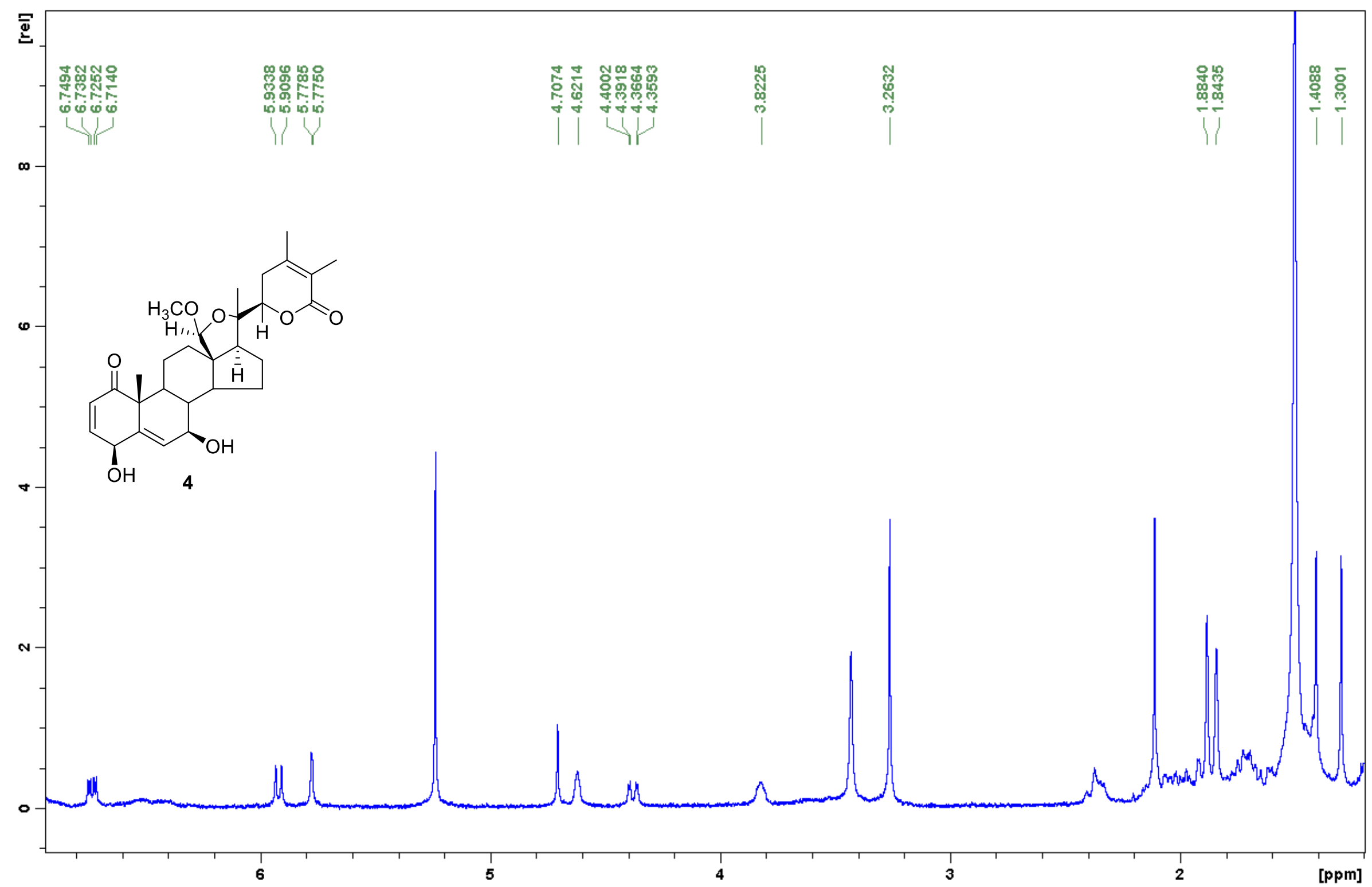


Figure S11. COSY spectrum of compound $4\left(\mathrm{CDCl}_{3}, 400.13 \mathrm{MHz}\right)$.

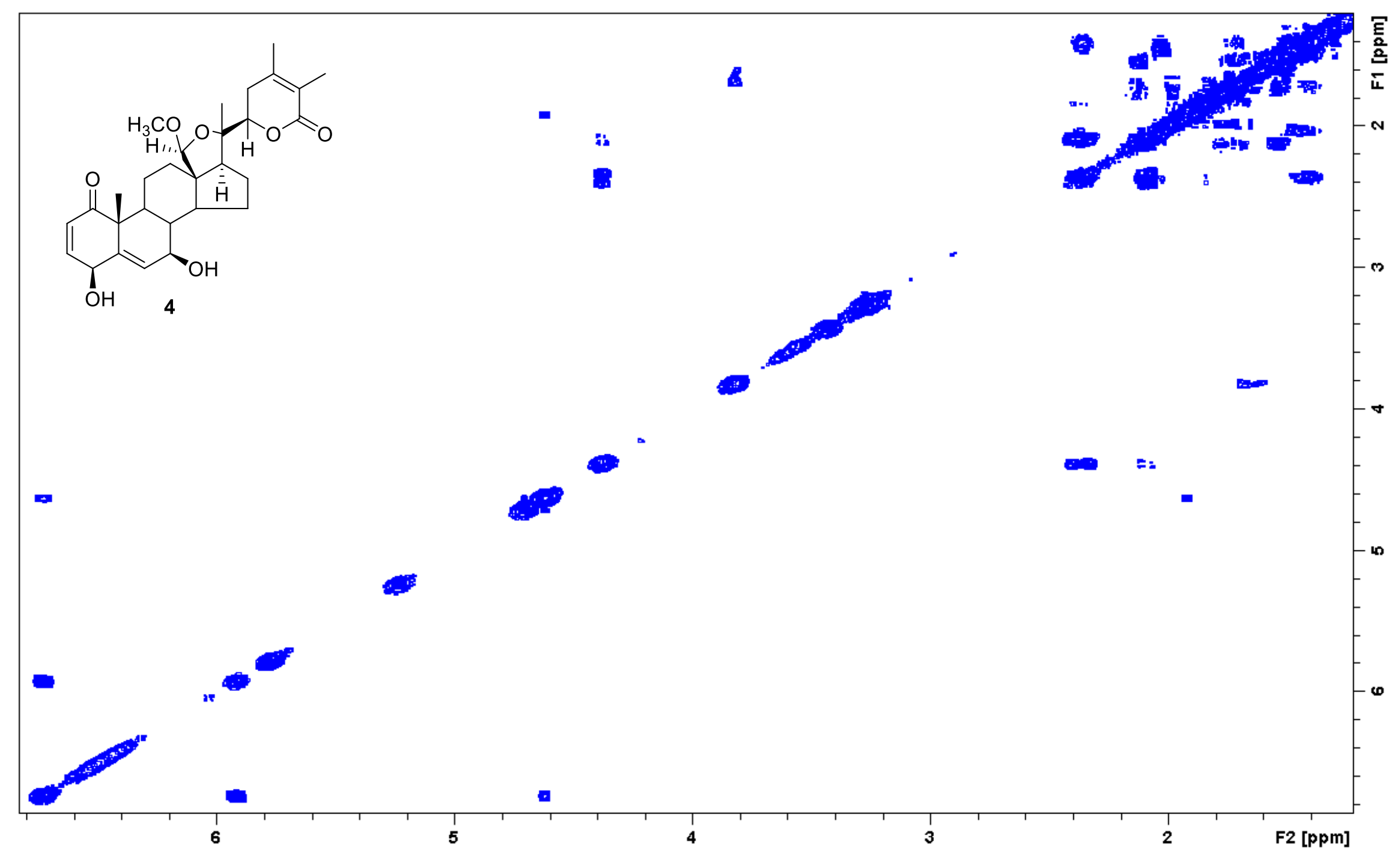


Figure S12. HSQC spectrum of compound $4\left(\mathrm{CDCl}_{3}, 400.13 \mathrm{MHz}\right)$.

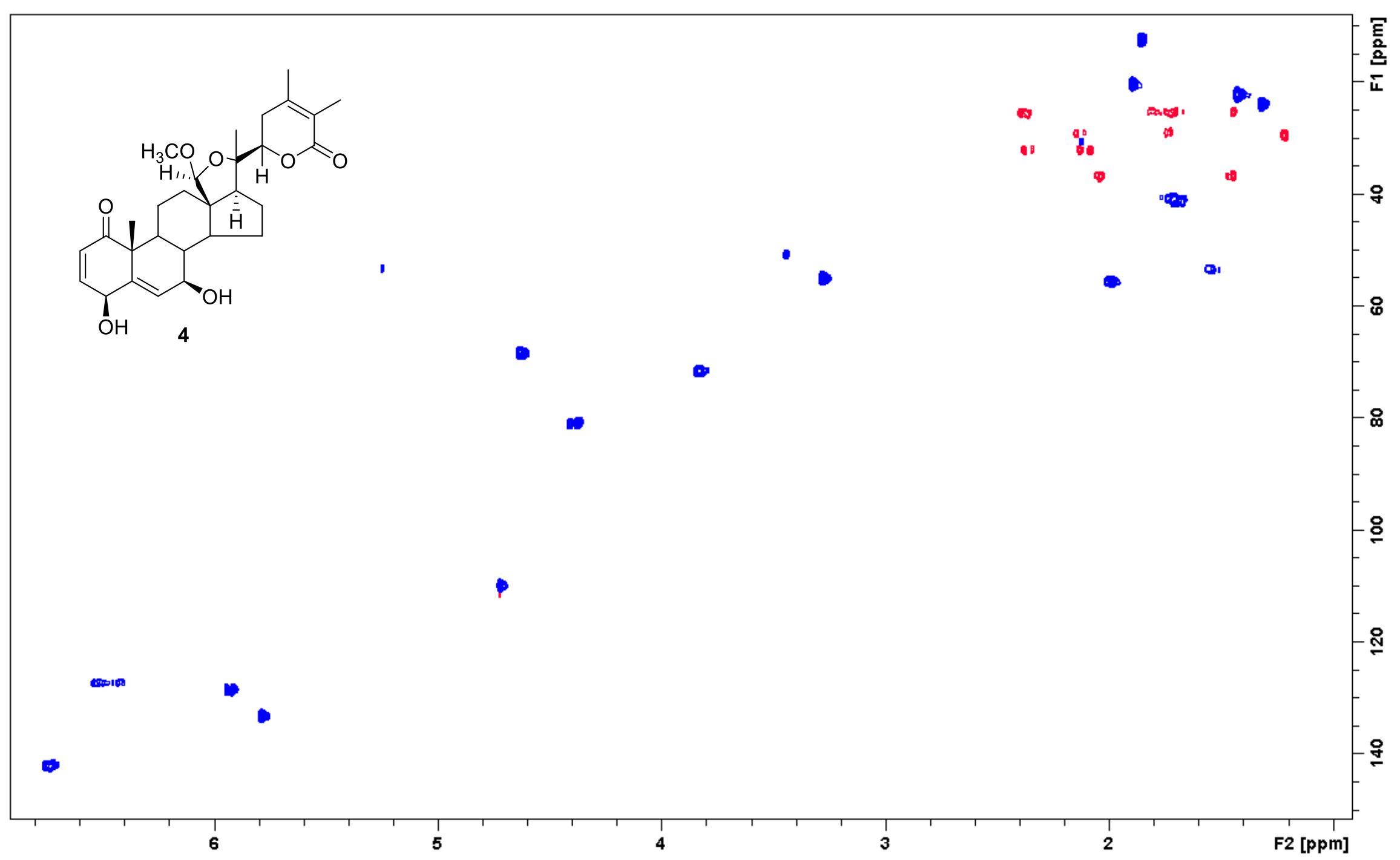


Figure S13. HMBC spectrum of compound $4\left(\mathrm{CDCl}_{3}, 400.13 \mathrm{MHz}\right)$.

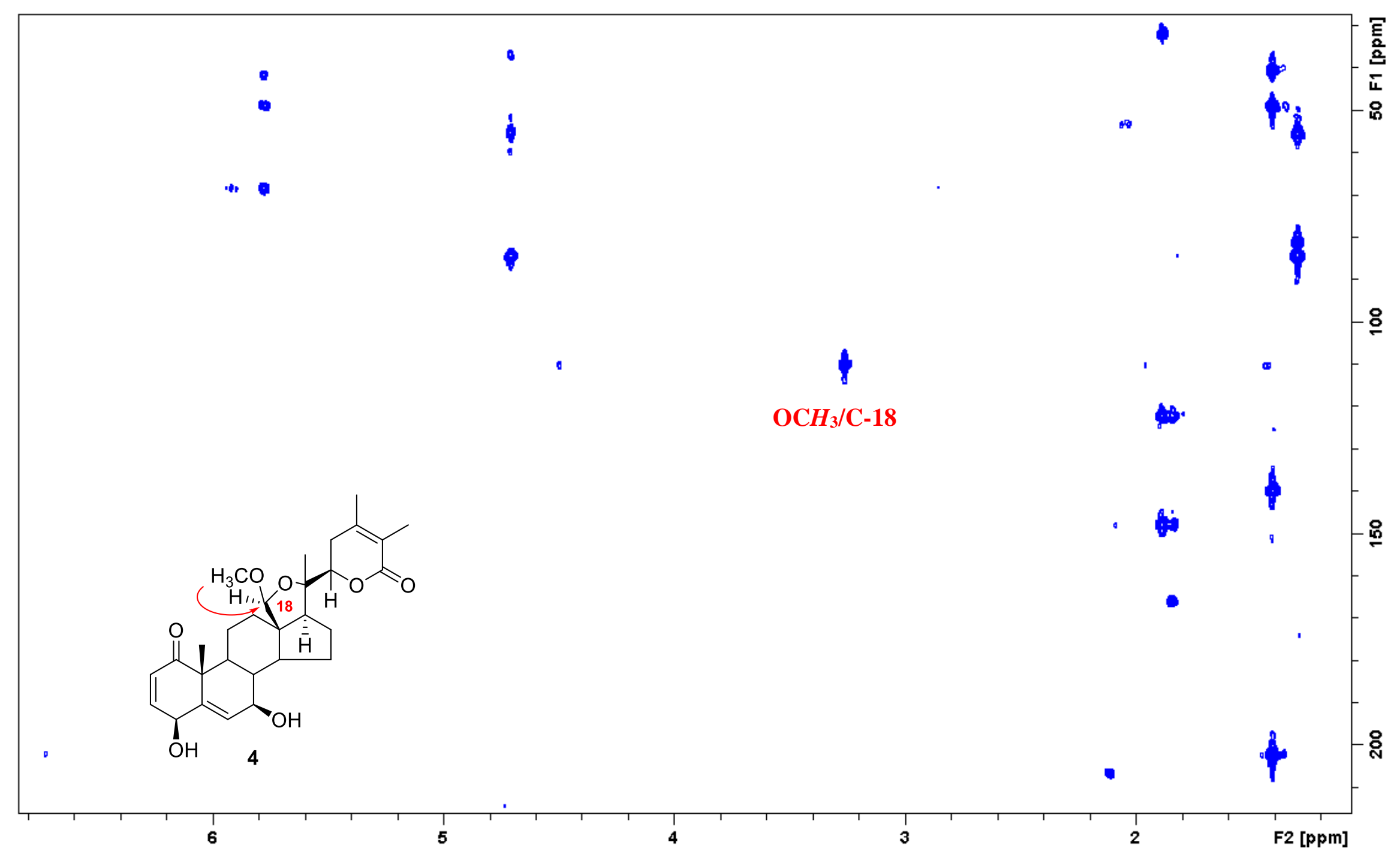


Figure S14. ${ }^{1} \mathrm{H}$ NMR spectrum of compound $5\left(\mathrm{CDCl}_{3}, 400.13 \mathrm{MHz}\right)$.

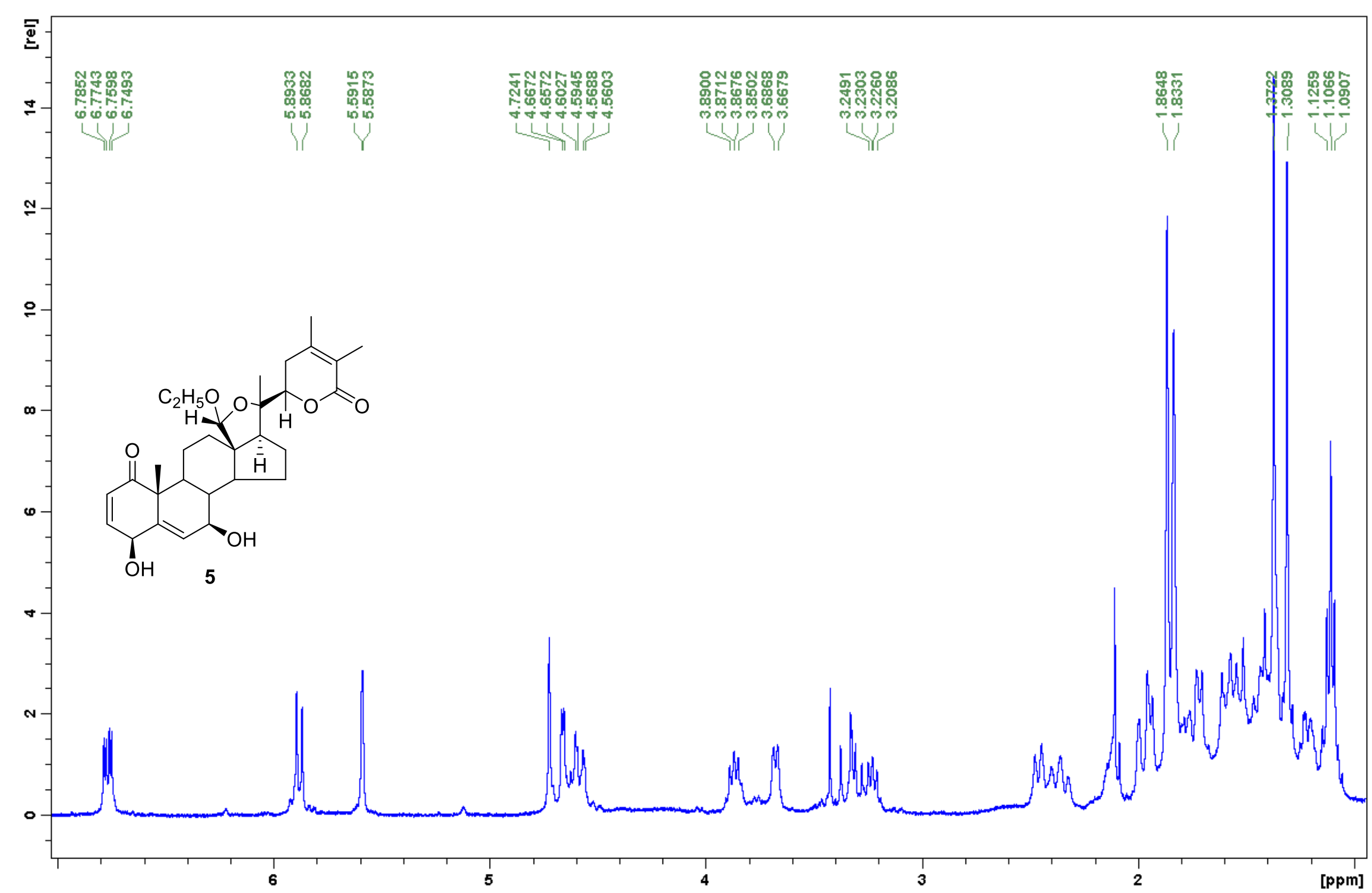


Figure S15. COSY spectrum of compound $\mathbf{5}\left(\mathrm{CDCl}_{3}, 400.13 \mathrm{MHz}\right)$.

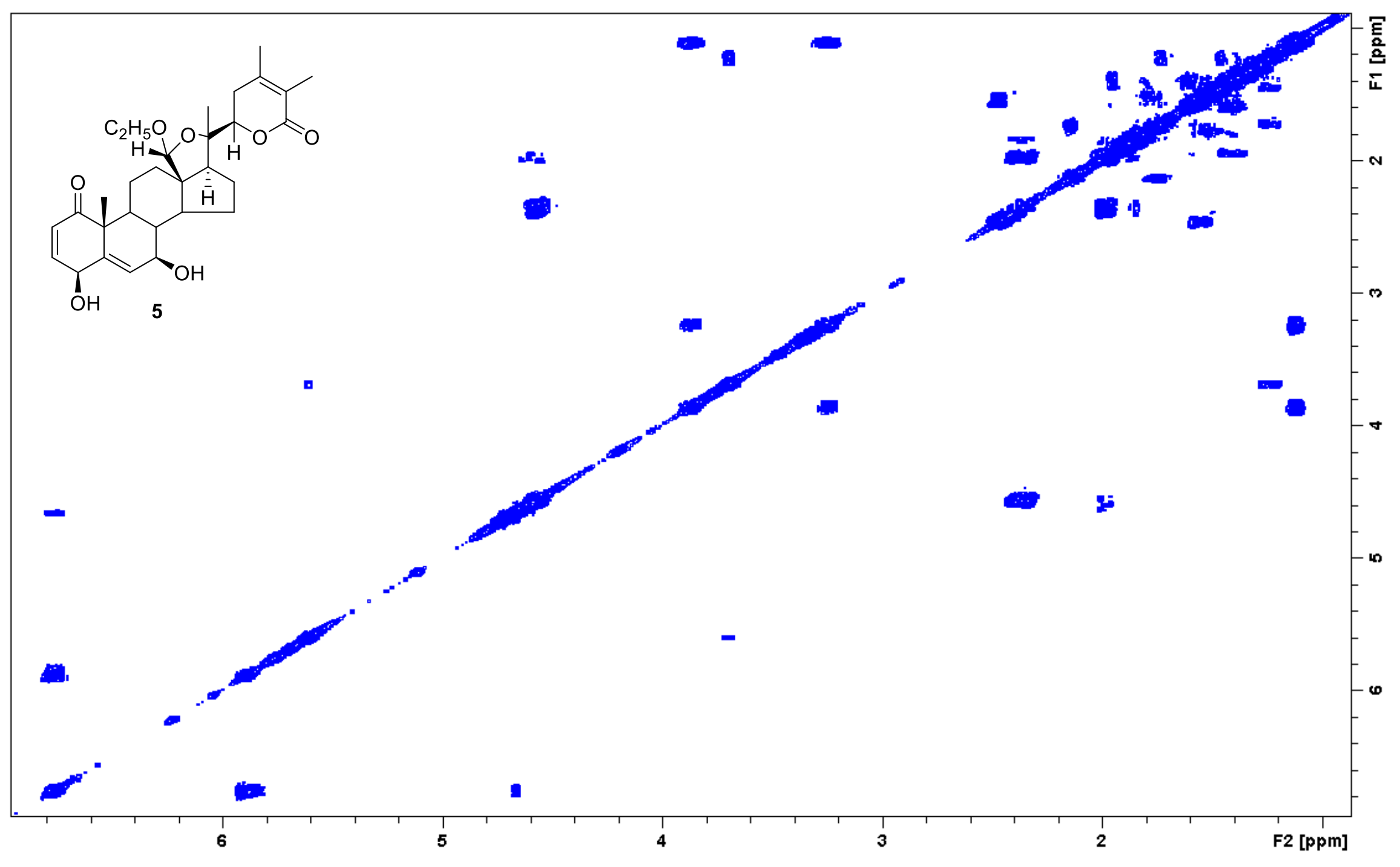


Figure S16. HSQC spectrum of compound $\mathbf{5}\left(\mathrm{CDCl}_{3}, 400.13 \mathrm{MHz}\right)$.

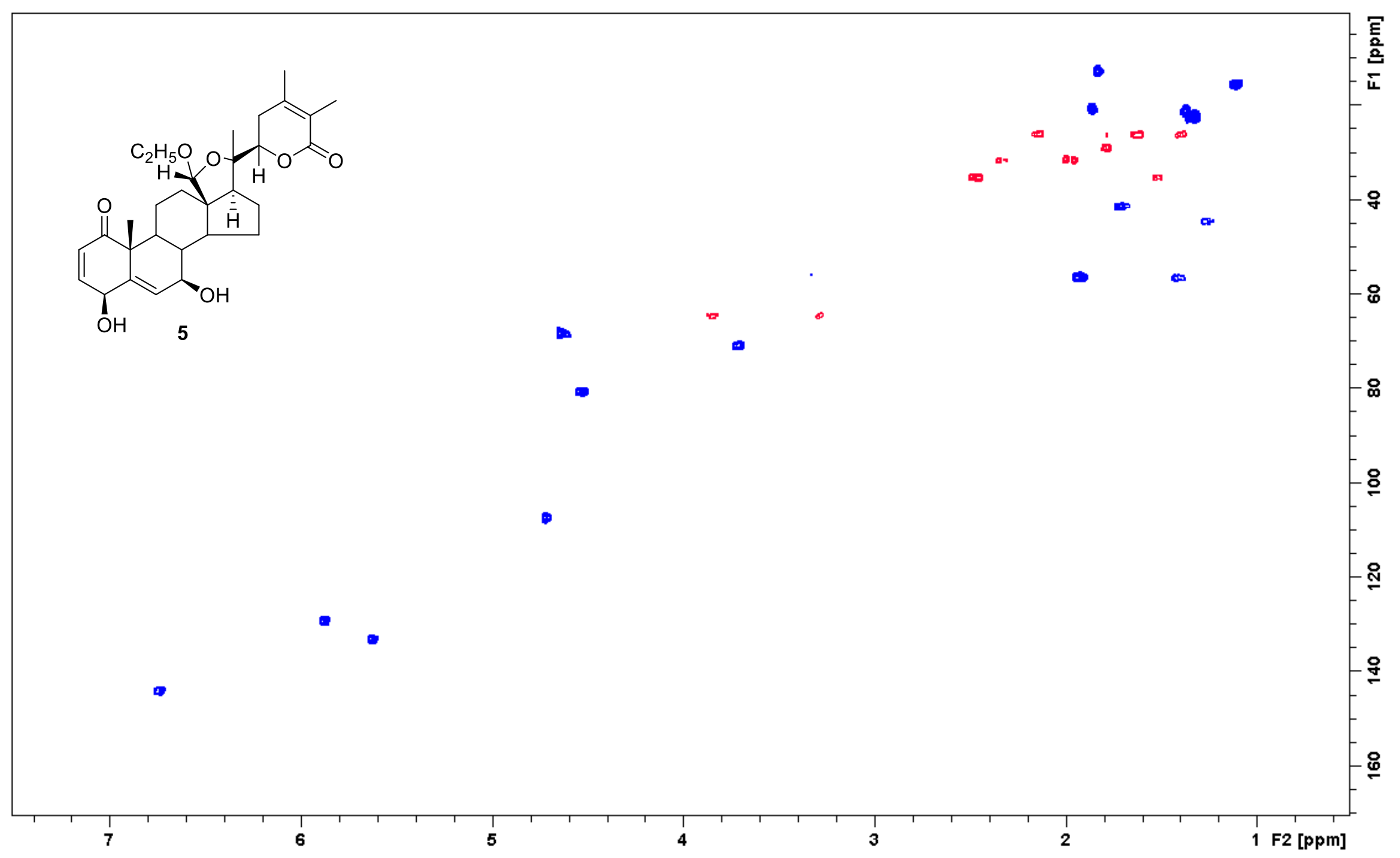


Figure S17. $\mathrm{HMBC}$ spectrum of compound $5\left(\mathrm{CDCl}_{3}, 400.13 \mathrm{MHz}\right)$.

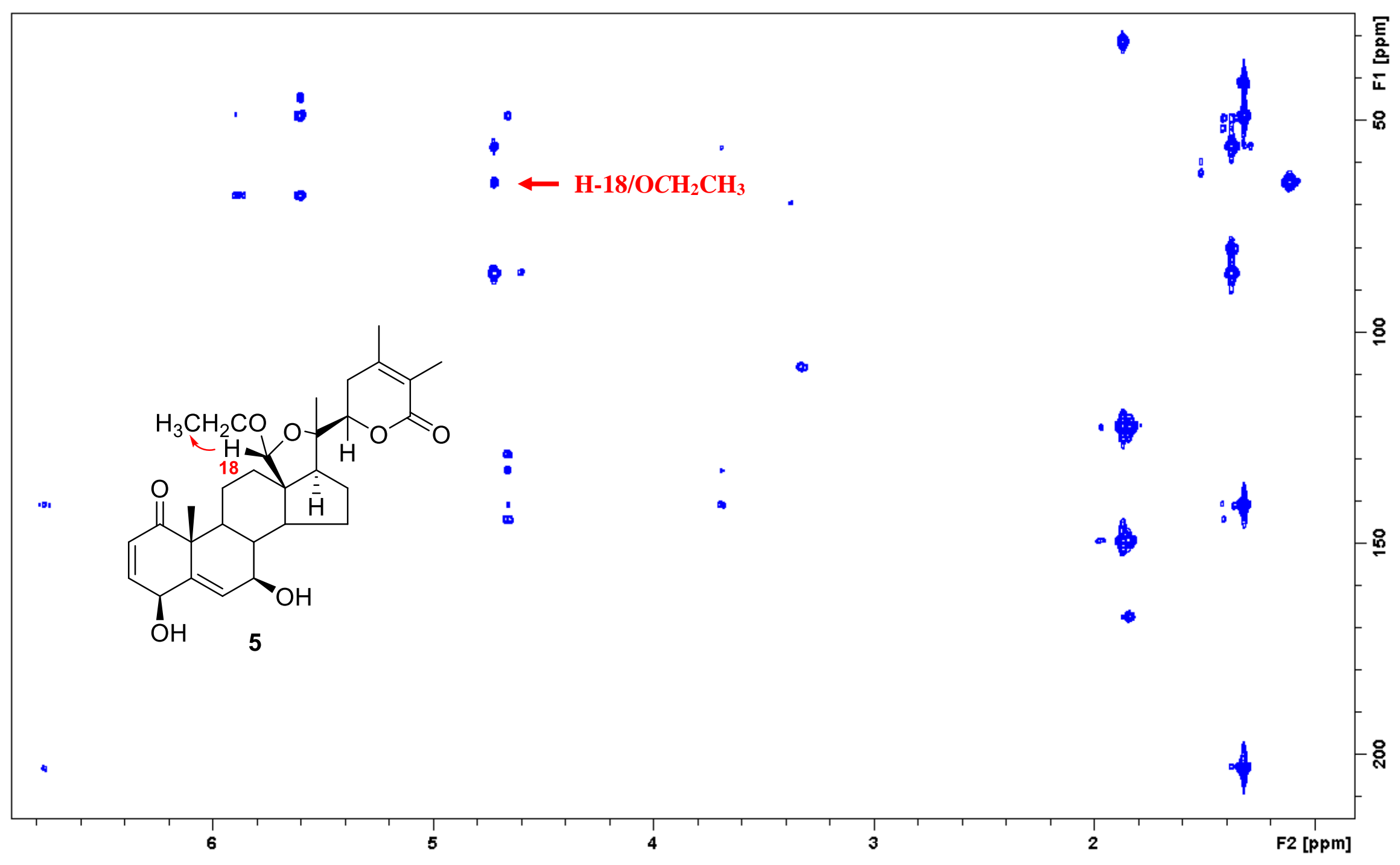


Figure S18. Relevant NOE correlations of compounds 4 and $5\left(\mathrm{CDCl}_{3}, 400.13 \mathrm{MHz}\right)$.
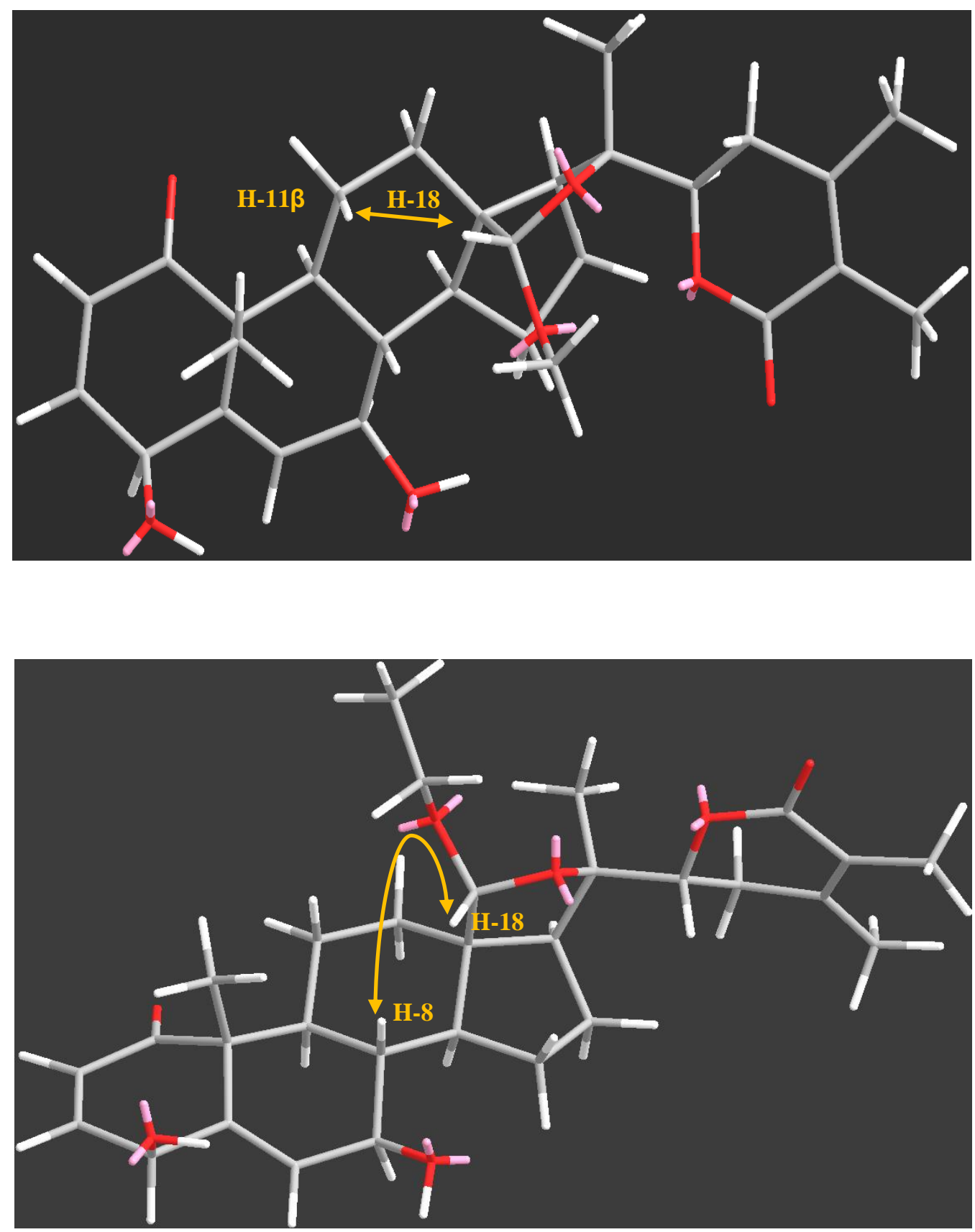
Selective Antiproliferative Withanolides from Species in the Genera Eriolarynx and Deprea. Castro et al.

Figure S19. GI50 range plot against tested human solid cell lines (cisplatin: blue bars, 1-13 yellow bars) and $\mathrm{GI}_{50}$ against human fibroblasts (red bars).

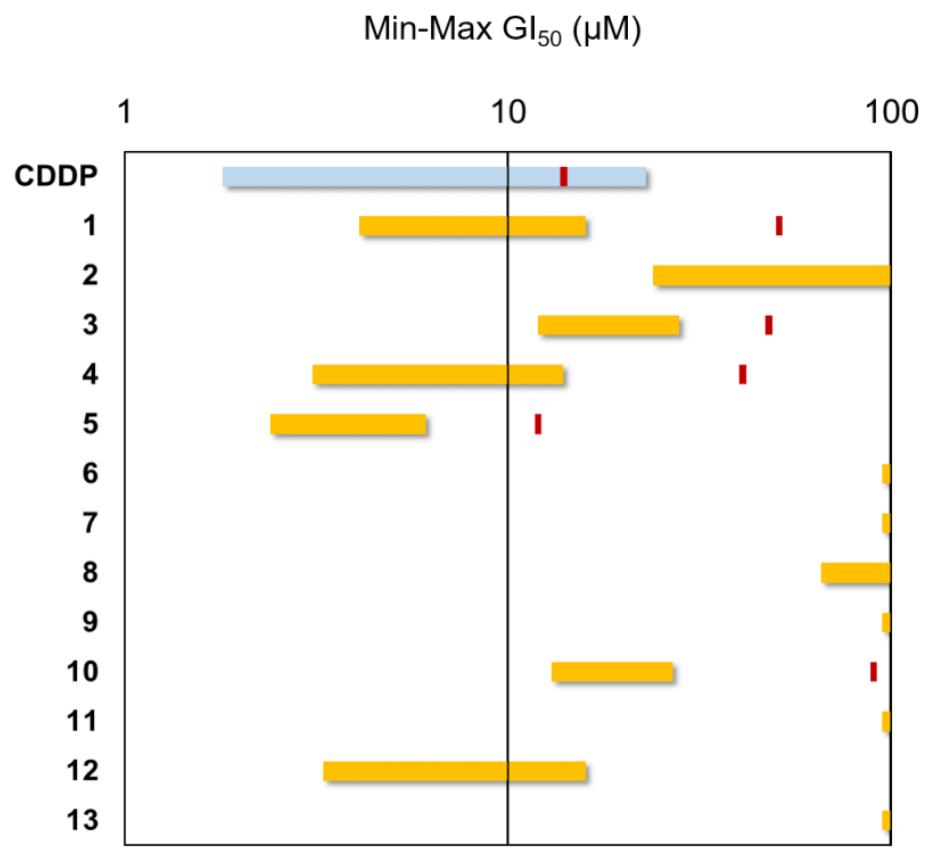

